\title{
Interazione: modificazione dell'associazione tra esposizione ed esito al variare di un terzo fattore
}

Giornale di Tecniche Nefrologiche e Dialitiche 2019, Vol. 3I (I) 40-42

(C) The Author(s) 2019

Article reuse guidelines:

sagepub.com/journals-permissions DOI: 10.1 I 177/0394936219836658

journals.sagepub.com/home/gtn

(S) AGE

\section{Francesco Franco e Anteo Di Napoli}

\begin{abstract}
Interaction: modification of the association between an exposure and outcome on the variation of a third factor

From a statistical perspective, interaction (effect modification) occurs when the effect of an exposure on an outcome depends on the level of another factor. In epidemiology, effect modification (interaction) occurs if the joint effect of two (or more) factors is different from the expected effect if considering only their independent effects. In an additive model, the effect of one exposure is added to effect of another exposure, and there is interaction if the joint effect of the two exposures together is greater than the sum of their individual effects. In a multiplicative model, the effect of the second exposure multiplies the effect of the first exposure, and there is interaction if the joint effect of the two exposures together is greater than the product of their individual effects. Interaction of two (or more) factors is synergic or antagonistic if the total effect is, respectively, greater or smaller than the sum of the individual effects of each factor.
\end{abstract}

\section{Keywords}

Interaction, Effect modification, Synergism, Antagonism
Con il presente lavoro si conclude la serie degli articoli dedicati ai tre temi fondamentali nell'ambito dell'inferenza causale: il bias (distorsione) ${ }^{1}$, il confondimento ${ }^{2}$ e l'interazione (modificazione di effetto). Si ha interazione quando l'azione di un fattore, indipendente sia dall'esposizione che dall'outcome in esame, modica l'effetto di un altro fattore (esposizione).

Iniziamo l'argomentazione col dare alcune definizioni relative al concetto di interazione. In termini statistici si parla di interazione quando l'effetto di un fattore su di un outcome dipende dai livelli di un altro fattore e, molto spesso, tale fenomeno prende anche il nome di eterogeneità ${ }^{3}$. In epidemiologia con il termine modificazione d'effetto (interazione) s'intende il fenomeno per cui uno o più fattori modificano significativamente la forza o la direzione dell'associazione tra il fattore di esposizione e l'esito in studio. In base alla definizione di MacMahon si ha interazione se il tasso di incidenza di una malattia, in presenza di due o più fattori di rischio, differisce dal tasso di incidenza che ci si attenderebbe dalla combinazione dei loro effetti individuali.

Se presente, l'effetto dell'interazione va sempre considerato in fase di analisi dei dati e deve essere accuratamente spiegato ${ }^{4}$.

La figura 1 rappresenta in modo diagrammatico una relazione causale in presenza di un fattore di interazione.

Una volta stabilita l'esistenza di un'associazione fra esposizione ed outcome, non dovuta alla presenza di fattori di confondimento (associazione causale), ci si deve chiedere: la forza dell'associazione rimane uguale in ciascuno degli strati che si formano sulla base di un terzo fattore (potenziale modificatore di effetto)? Se la forza

Comitato Editoriale GTND, Italy

Corrispondenza:

Francesco Franco, Comitato Editoriale GTND, Italy.

E-mail: franco_francesco@fastwebnet.it 
dell'associazione rimane uguale all'interno degli strati del terzo fattore, non si ha interazione; se invece differisce, si è in presenza di un fattore modificatore di effetto. Ma come interagiscono più fattori nel causare una patologia?

Va detto, preliminarmente, che esistono due tipi di interazione: l'interazione positiva (o sinergismo) in cui

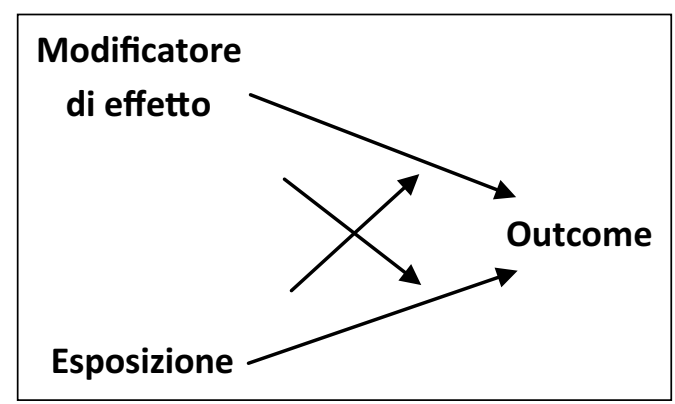

Figura I. Interazione (modificazione di effetto). l'effetto del fattore di esposizione sull'outcome è potenziato dalla presenza/livello di un altro fattore, e l'interazione negativa (o antagonismo) in cui l'effetto del fattore di esposizione sull'outcome diminuisce in presenza del modificatore di effetto.

L'affermazione dell'esistenza o meno di un'interazione dipende dalla misura di effetto presa in considerazione ${ }^{5} \mathrm{e}$ quindi dal tipo di modello assunto nell'analisi dei dati:

- Rischio Attribuibile (modello additivo)

- Rischio Relativo (modello moltiplicativo)

La scelta del tipo di modello assunto dipende da considerazioni sulla biologia e il meccanismo patogenetico alla base della patologia in esame, nonché dall'azione esercitata dai fattori di esposizione nei diversi contesti.

In un modello additivo, in presenza di più fattori di rischio indipendenti, l'effetto totale esercitato dai fattori stessi sarà dato dalla somma dei singoli fattori di rischio.

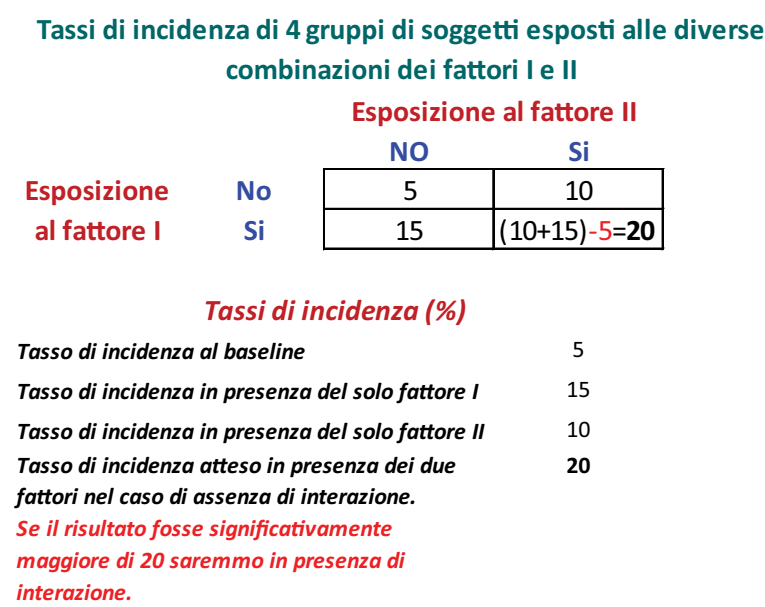

5

Tasso di incidenza in presenza del solo fattore I $\quad 15$

Tasso di incidenza in presenza del solo fattore II $\quad 10$ Tasso di incidenza atteso in presenza dei due $\quad 20$ fattori nel caso di assenza di interazione.

Se il risultato fosse significativamente

maggiore di 20 saremmo in presenza di interazione.

Rischi attribuibili ai fattori I e II nelle quattro diverse combinazioni

Esposizione al fattore II

Esposizione al fattore I

\begin{tabular}{c|c|c|}
\multicolumn{1}{c}{} & \multicolumn{1}{c}{ NO } & \multicolumn{1}{c}{ Si } \\
\cline { 2 - 3 } No & 0 & 5 \\
\cline { 2 - 3 } Si & 10 & 15 \\
\cline { 2 - 3 }
\end{tabular}

Il fattore I aumenta il tasso di incidenza dell'esito in esame di 10 punti percentuali sia in assenza che in presenza del fattore II (stessa forza negli strati del fattore II).

II fattore II aumenta il tasso di incidenza dell'esito in esame di 5 punti percentuali sia in assenza che in presenza del fattore I (stessa forza negli strati del fattore I).

Figura 2. Modello Additivo (misura dell'effetto: rischio attribuibile). Esempio liberamente tratto dal libro di Gordis L. Epidemiology [6].

\begin{tabular}{|c|c|c|c|c|c|c|c|}
\hline \multicolumn{4}{|c|}{$\begin{array}{c}\text { Tassi di incidenza di } 4 \text { gruppi di soggetti esposti alle diverse } \\
\text { combinazioni dei fattori I e II }\end{array}$} & \multicolumn{4}{|c|}{$\begin{array}{l}\text { Rischi Relativi dei fattori I e II nelle quattro } \\
\text { diverse combinazioni }\end{array}$} \\
\hline \multirow{4}{*}{$\begin{array}{l}\text { Esposizione } \\
\text { al fattore I }\end{array}$} & & \multicolumn{2}{|c|}{ Fattore II } & \multicolumn{4}{|c|}{ Esposizione al fattore II } \\
\hline & & NO & $\mathrm{Si}$ & \multirow{3}{*}{$\begin{array}{l}\text { Esposizione } \\
\text { al fattore I }\end{array}$} & \multirow{3}{*}{$\begin{array}{l}\text { No } \\
\text { Si }\end{array}$} & NO & $\mathrm{Si}$ \\
\hline & No & 5 & 10 & & & 1 & 2 \\
\hline & $\mathrm{Si}$ & 15 & $5 \times 2 \times 3=30$ & & & 3 & 6 \\
\hline \multicolumn{4}{|c|}{ Tassi di incidenza (\%) } & \multirow{4}{*}{\multicolumn{4}{|c|}{$\begin{array}{l}\text { II fattore I triplica il tasso di incidenza dell'esito in esame sia in assenza } \\
\text { che in presenza del fattore II (stessa forza negli strati del fattore II). } \\
\text { II fattore II raddoppia il tasso di incidenza dell'esito in esame sia in } \\
\text { assenza che in presenza del fattore I (stessa forza negli strati del fattore I) }\end{array}$}} \\
\hline \multicolumn{3}{|c|}{ Tasso di incidenza al baseline } & \multirow{2}{*}{$\begin{array}{c}5 \\
15\end{array}$} & & & & \\
\hline \multicolumn{3}{|c|}{ Tasso di incidenza in presenza del solo fattore I } & & & & & \\
\hline \multicolumn{3}{|c|}{ Tasso di incidenza in presenza del solo fattore II } & $\begin{array}{l}15 \\
10\end{array}$ & & & & \\
\hline $\begin{array}{l}\text { Tasso di inciden } \\
\text { fattori nel caso } \\
\text { Se il risultato fo } \\
\text { maggiore di } 30 \\
\text { interazione. }\end{array}$ & $\begin{array}{l}\text { atteso in } \\
\text { assenza } \\
\text { signific } \\
\text { emmo ir }\end{array}$ & $\begin{array}{l}\text { dei due } \\
\text { one. } \\
\text { te } \\
\text { di }\end{array}$ & 30 & & & & \\
\hline
\end{tabular}

Figura 3. Modello Moltiplicativo (misura dell'effetto: rischio relativo). Esempio liberamente tratto dal libro di Gordis L. Epidemiology [6]. 
Se l'effetto totale risultasse maggiore della somma dei singoli fattori si sarebbe di fronte ad un fenomeno di interazione (sinergismo).

In un modello moltiplicativo, l'effetto totale sarà dato, invece, dal prodotto dei singoli fattori di rischio. Se l'effetto totale risultasse maggiore del prodotto dei singoli fattori si sarebbe in presenza di interazione.

La maggior parte dei ricercatori ritiene che se l'effetto congiunto di due fattori produce un Rischio Attribuibile maggiore di quello ottenibile sotto l'ipotesi di un modello additivo, è prova sufficiente di interazione positiva (sinergismo). ${ }^{6}$

Di seguito si mostrano due esempi (figura 2 e figura 3 ) relativi ai contesti di un modello additivo e di un modello moltiplicativo.

Nell'esempio di modello additivo (figura 2), il fattore I aumenta il tasso di incidenza dell'esito in esame di 10 punti percentuali sia in assenza che in presenza del fattore II (stessa forza negli strati del fattore II); il fattore II aumenta il tasso di incidenza dell'esito in esame di 5 punti percentuali sia in assenza che in presenza del fattore I (stessa forza negli strati del fattore I). Il tasso di incidenza atteso in presenza dei due fattori, nel caso di assenza di interazione, sarebbe pari al $20 \%$. Se il risultato fosse significativamente maggiore di 20 saremmo in presenza di interazione.

Nell'esempio di modello moltiplicativo (figura 3), il fattore I triplica il tasso di incidenza dell'esito in esame sia in assenza che in presenza del fattore II (stessa forza negli strati del fattore II); il fattore II raddoppia il tasso di incidenza dell'esito in esame sia in assenza che in presenza del fattore I (stessa forza negli strati del fattore I). Il tasso di incidenza atteso in presenza dei due fattori nel caso di assenza di interazione sarebbe pari al $30 \%$. Se il risultato fosse significativamente maggiore di 30 saremmo in presenza di interazione.

\section{Dichiarazione di assenza di conflitto di interessi}

Gli Autori dichiarano di non avere conflitti di interessi.

\section{Finanziamenti}

Gli Autori dichiarano di non aver ricevuto finanziamenti specifici da qualsiasi ente nei settori pubblico, privato o senza fini di lucro.

\section{Bibliografia}

1. Franco F and Di Napoli A. Il bias: una insidia per la validità di uno studio [Bias: a pitfall to the validity of a study]. G Tec Nefrol Dial. 2018;. doi:10.1177/0394936218807904.

2. Franco F and Di Napoli A. Il confondimento: un bias della stima dell'effetto fra una esposizione ed un esito di salute [Confounding: a bias in the estimation effect between exposure and health outcome]. G Tec Nefrol Dial. (in press)

3. Greenland S. Effect Modification and Interaction. Wiley StatsRef: Statistics Reference Online https://doi.org/10.1002 /9781118445112.stat03728. (ultimo accesso 14 marzo 2019)

4. Lopalco PL and Tozzi AE. Epidemiologia facile. Roma: Il Pensiero Scientifico Editore 2003; 7:51-4.

5. Rothman KJ, Greenland $\mathrm{S}$ and Lash TL. Modern epidemiology. Third ed. Philadelphia: Lippincott Williams \& Wilkins 2008; 4:61-2.

6. Gordis L. Epidemiology. Fourth ed. Philadelphia: Elsevier Saunders 2014; 11:256-61. 